\title{
Os cuidados pré-natais no manejo da pré-eclâmpsia
}

\author{
Prenatal care in the management of pre eclampsia \\ Atención prenatal em el tratamiento de la preeclampsia
}

Recebido: 18/09/2021 | Revisado: 23/09/2021 | Aceito: 24/09/2021 | Publicado: 26/09/2021

\author{
Marcello Vieira dos Santos \\ ORCID: https://orcid.org/0000-0002-3356-1619 \\ Centro Universitário do Estado do Pará, Brasil \\ E-mail: marcellosantos277@gmail.com \\ Cassiane da Silva Portela Pinto \\ ORCID: https://orcid.org/0000-0002-5732-8555 \\ Universidade do Estado do Pará, Brasi \\ E-mail:cassiane_portela@yahoo.com \\ Camila Cristina Girard Santos \\ ORCID: https://orcid.org/0000-0003-3159-7041 \\ Universidade do Estado do Pará, Brasil \\ E-mail: camilagirard@hotmail.com
}

\begin{abstract}
Resumo
A Pré-Eclâmpsia (PE) é definida como uma doença específica da gestação associadas ao aparecimento de hipertensão com caráter multissistêmico ou multifatorial após a $20^{\mathrm{a}}$ semana de gestação, possuindo altos índices de morbidade, mortalidade e prematuridade e é responsável por $10 \%$ a $15 \%$ dos casos de morte maternas no mundo. Nesse sentido, faz-se necessário fornecer assistência à gestante o mais precocemente possível, garantindo a saúde materna e fetal de forma humanizada, integral e de qualidade. Nesse interim, o presente trabalho trata-se de uma revisão da literatura e tem como objetivo propor uma reflexão a partir da análise de estudos científicos acerca dos cuidados pré-natais no manejo da PE. Não obstante, verificou-se que $61,2 \%$ das gestantes não realizam o pré-natal, favorecendo desfechos obstétricos negativos como abortamento, eclampsia, hemorragias, prematuridade e descolamento prematuro de placenta. Ademais, diversos fatores estão associados ao aparecimento da PE como estresse, ansiedade, sentimento de abandono em decorrência de muitas mulheres não terem a participação do cônjuge no pré-natal, assim dificultando sua adesão. Dessa maneira, cabe aos profissionais da saúde o papel de sensibilizar e informar as gestantes sobre os riscos, cuidados, uso de medicação, alimentação, orientações sobre as consultas, além de manter uma comunicação direta e ativa durante toda a assistência Nesse contexto, faz-se necessário que a gestante tenha um acompanhamento multiprofissional durante a sua assistência pré-natal, visando um atendimento holístico que supra as suas necessidades nutricionais, físicas e clínicas durante todo o período gestacional.
\end{abstract}

Palavras-chave: Gestante; Pré-eclâmpsia; Cuidados pré-natais; Parto; Modelos de assistência à saúde.

\begin{abstract}
Pre-eclampsia (PE) is defined as a pregnancy-specific disease associated with the onset of hypertension with a multisystemic or multifactorial character after the 20th week of pregnancy, with high rates of morbidity, mortality and prematurity and is responsible for $10 \%$ to $15 \%$ of maternal death cases in the world. In this sense, it is necessary to provide assistance to pregnant women as early as possible, ensuring maternal and fetal health in a humanized, comprehensive and quality way. In the meantime, the present work is a literature review and aims to propose a reflection based on the analysis of scientific studies on prenatal care in the management of PE. Nevertheless, it was found that $61.2 \%$ of pregnant women do not undergo prenatal care, favoring negative obstetric outcomes such as abortion, eclampsia, hemorrhages, prematurity and placental abruption. Furthermore, several factors are associated with the onset of PE, such as stress, anxiety, feeling of abandonment due to the fact that many women do not have their spouse's participation in prenatal care, thus hindering their adherence. Thus, it is up to health professionals the role of sensitizing and informing pregnant women about the risks, care, use of medication, food, guidance on consultations, in addition to maintaining direct and active communication throughout the assistance. if necessary, the pregnant woman has a multidisciplinary follow-up during her prenatal care, aiming at a holistic care that meets her nutritional, physical and clinical needs throughout the gestational period.
\end{abstract}

Keywords: Pregnant; Pre eclampsia; Prenatal care; Childbirth; Health care models.

\section{Resumen}

La preeclampsia (EP) se define como una enfermedad específica del embarazo asociada a la aparición de hipertensión de carácter multisistémico o multifactorial a partir de la semana 20 de gestación, con altas tasas de morbilidad, mortalidad y prematuridad y es responsable de un $10 \%$ a $15 \%$ de los casos de muerte materna en el mundo. En este 
sentido, es necesario brindar asistencia a las mujeres embarazadas lo antes posible, asegurando la salud materna y fetal de manera humanizada, integral y de calidad. Mientras tanto, el presente trabajo es una revisión de la literatura y tiene como objetivo proponer una reflexión basada en el análisis de estudios científicos sobre el cuidado prenatal en el manejo de la EP. Sin embargo, se encontró que el 61,2\% de las gestantes no reciben atención prenatal, lo que favorece resultados obstétricos negativos como aborto, eclampsia, hemorragias, prematuridad y desprendimiento de placenta. Además, varios factores se asocian con la aparición de la EP, como el estrés, la ansiedad, el sentimiento de abandono por el hecho de que muchas mujeres no cuentan con la participación de su cónyuge en la atención prenatal, lo que dificulta su adherencia. Así, corresponde a los profesionales de la salud el rol de sensibilizar e informar a la gestante sobre los riesgos, cuidados, uso de medicación, alimentación, orientación en las consultas, además de mantener una comunicación directa y activa durante toda la asistencia. De ser necesario, la gestante tiene un seguimiento multidisciplinario durante su atención prenatal, con el objetivo de una atención integral que satisfaga sus necesidades nutricionales, físicas y clínicas durante todo el período gestacional.

Palabras clave: Embarazada; Preeclampsia; Cuidado prenatal; Parto; Modelos de atención médica.

\section{Introdução}

A Pré-Eclâmpsia (PE) é definida como uma doença específica da gestação associadas ao aparecimento de hipertensão com caráter multissistêmico ou multifatorial após a 20ª semana de gestação (Kahhale, Francisco \& Zugaib, 2018; Febrasgo, 2019). Por ser multifatorial, geralmente o diagnóstico de PE está ligado à pressão arterial sistémica durante a gravidez $\geq 140$ $\mathrm{mmHg}$ e/ou diastólica $\geq 90 \mathrm{mmHg}$, junto a um quadro proteinúrico ou, com menos frequência, disfunção no órgão alvo (Febrasgo, 2019; Sociedade Brasileira de Cardiologia, 2021).

Ademais, a PE pode ser dividida fisiopatologicamente em "placentária" quando o problema se origina na placenta e "materna", de origem endotelial difusa (Guida, 2017). Outra classificação gira em todo do período de instalação da doença, que pode ser "precoce", quando a doença se inicia até a $34^{\mathrm{a}}$ semana gestacional e "tardia", com início após a $34^{\mathrm{a}}$ semana (Guida, 2017).

Por outro lado, caso não seja tratada, a PE possui risco de evolução para casos mais graves, como: Pré-Eclâmpsia Grave (PEG), eclampsia, síndrome de HELLP, hipertensão arterial crônica, coagulação intravascular disseminada, insuficiência renal, edema agudo de pulmão, hemorragia cerebral e até na morte (Kahhale, Francisco \& Zugaib, 2018; Oldra et al., 2021).

À vista disso, de acordo com a Organização Mundial da Saúde (OMS) a PE possui altos índices de morbidade, mortalidade e prematuridade e é responsável por $10 \%$ a $15 \%$ dos casos de morte maternas diretas no mundo, visto que a probabilidade de morte materna em decorrência da PE é de 11,36 vezes maior se comparada a mães sem PE (Fundação Oswaldo Cruz, 2018; Febrasgo, 2019). Além disso, a patologia de estudo também é causadora da morte indireta de mais de 500 mil bebês por ano (Governo de Santa Catarina, 2019).

Outrossim, o Brasil, em 2019, teve 1.576 mortes maternas, dos quais 118 (7,48\%) ocorreram devido à hipertensão materna com a presença de proteinúria, segundo o banco de dados do departamento de informática do Sistema Único de Saúde (DATASUS).

Mediante isso, faz-se necessário fornecer a assistência à gestante o mais precocemente possível, a fim de garantir a saúde materna e fetal e evitar afecções durante a gestação e no momento do parto (Marques et al., 2020; Fassarella et al., 2020). Nesse sentido, uma assistência pré-natal bem estruturada garante durante todo o processo gestacional a promoção da saúde, além da prevenção, diagnóstico e tratamento prévio de doenças (Nascimento et al., 2021).

Além disso, os cuidados pré-natais constituem ações assistencialista de forma humanizada, integral e de qualidade, que vai desde o início da gravidez até o pós-parto, englobando os cuidados com a criança (Santos \& Batista, 2018; Mendes et al., 2020).Nessa perspectiva, o Ministério da Saúde implementou o Programa de Humanização no Pré-Natal e Nascimento (PHPN) em junho de 2000, tendo como principal estratégia um cuidado humanizado e que incentive ações visando a redução de complicações obstétricas como a pré-eclâmpsia (Silva, 2020) 
Nesse ínterim, é indubitável a necessidade de iniciar precocemente o pré-natal para a detecção precoce de patologias hipertensivas gestacionais, como a pré-eclâmpsia, que causam um maior risco à gestação e que necessitam de um maior acompanhamento (Fassarella et al., 2020). Consequentemente, é importante enquadrar essa gestante no pré-natal de alto risco e assim aumentar a frequência das visitas pré-natais para um melhor monitoramento da saúde materna e fetal, além de implementar as medidas profiláticas adequadas à paciente, visando a melhora do desfecho obstétrico (Oliveira et al., 2021; Marques et al., 2020).

Ademais, é necessário que os cuidados pré-natais às gestantes que apresentam os fatores de risco da PE - idade, obesidade, histórico familiar, patologias pré-existentes e entre outros - seja iniciado imediatamente após o diagnóstico. (Fundação Oswaldo Cruz 2018; Fassarella et al., 2020). Diante disso, o objetivo desse trabalho é propor uma reflexão a partir da análise de estudos científicos acerca dos cuidados pré-natais no manejo da pré-eclâmpsia

\section{Metodologia}

O estudo trata-se de uma revisão de literatura cuja função envolva analisar, reunir e comparar investigações e informações prévias sobre uma dada temática, com o objetivo de propor reflexões e construção de novos estudos (Gonçalves, 2019). Esse método foi estruturado em 6 etapas, na primeira etapa foi definido a pergunta norteadora "Quais os cuidados prénatais no manejo da pré-eclâmpsia?”.

Na segunda etapa, foram catalogados estudos nas bases de dados Latino Americana e do Caribe em Ciências da Saúde (LILACS), National Library of Medicine (PUBMED), Scientific Electronic Library (Scielo) utilizando os seguintes descritores "Gestante", "Pré-eclâmpsia", "Cuidados pré-natais", "Parto" e "Modelos de Assistência à Saúde". Para a seleção dos estudos foram utilizados os critérios de inclusão: ano de 2016 a 2021 e estudos no idioma inglês, espanhol e português.

$\mathrm{Na}$ terceira etapa, foram selecionados os artigos que se encaixavam na pergunta norteadora. Nas duas etapas seguintes foram feitas análise e interpretação dos dados coletados, cuja verificou-se que os trabalhos possuíam uma vertente de análise acerca da mortalidade materna, intrauterina e neonatal, perfil das gestantes com PE, o papel da equipe multiprofissional na assistência pré-natal das gestantes com PE e os déficits na realização do cuidado pré-natal.

Por fim, a sexta etapa foi executado a condensação das informações e a exposição das informações encontradas em um fluxograma, que estão sinalizadas na Figura 1. 
Figura 1 - Fluxograma de seleção dos artigos sobre os cuidados pré-natais no manejo da pré-eclâmpsia.

\section{Estudos encontrados nas bases de dados:}

LILACS ( $\mathrm{n}$ 799): Palavras-chave: Gestante AND Pré-eclâmpsia $(\mathrm{n}=177)$; Pré-eclâmpsia AND Cuidados pré-natais $(\mathrm{n}=3)$; Gestante AND Cuidados Pré-natais ( $\mathrm{n}=3$ ); Parto AND Gestante ( $\mathrm{n}=307)$; Parto AND Modelo de Assistência à Saúde $(\mathrm{n}=143)$; cuidados pré-natais AND Parto ( $\mathrm{n}=18)$; Parto AND Pré-eclâmpsia $(\mathrm{n}=121)$; Modelo de Assistência à Saúde AND Gestante $(\mathrm{n}=$ 26); Modelo de Assistência à Saúde AND Pré-eclâmpsia ( $\mathrm{n}=1)$

PUBMED: $(\mathrm{n}=18)$ Palavras-chave: Gestante AND Pré-eclâmpsia $(\mathrm{n}=12)$; Pré-eclâmpsia AND Cuidados pré-natais $(\mathrm{n}=0)$; Gestante AND Cuidados Pré-natais $(n=0)$; Parto AND Gestante $(n=2)$; Parto AND Modelo de Assistência à Saúde $(n=0)$; cuidados pré-natais AND Parto $(n=0)$; Parto AND Pré-eclâmpsia $(n=4)$; Modelo de Assistência à Saúde AND Gestante $(n=0)$; Modelo de Assistência à Saúde AND Pré-eclâmpsia (n=0)

SCIELO ( $\mathrm{n}=214)$ Palavras-chave: Gestante AND Pré-eclâmpsia $(\mathrm{n}=29)$; Pré-eclâmpsia AND Cuidados pré-natais $(\mathrm{N}=0)$; Gestante AND Cuidados Pré-natais $(n=5)$; Parto AND Gestante $(n=93)$; Parto AND Modelo de Assistência à Saúde $(n=53)$; cuidados pré-natais AND Parto $(n=2)$; Parto AND Pré-eclâmpsia $(n=24)$; Modelo de Assistência à Saúde AND Gestante $(n=8)$; Modelo de Assistência à Saúde AND Pré-eclâmpsia $(\mathrm{n}=0)$

Total: $(\mathrm{n}=1.031)$

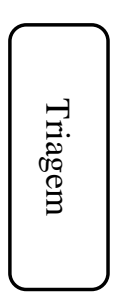

Estudos rastreados: Trabalhos publicados entre 2016 a 2021, disponíveis nos idiomas português, inglês e espanhol

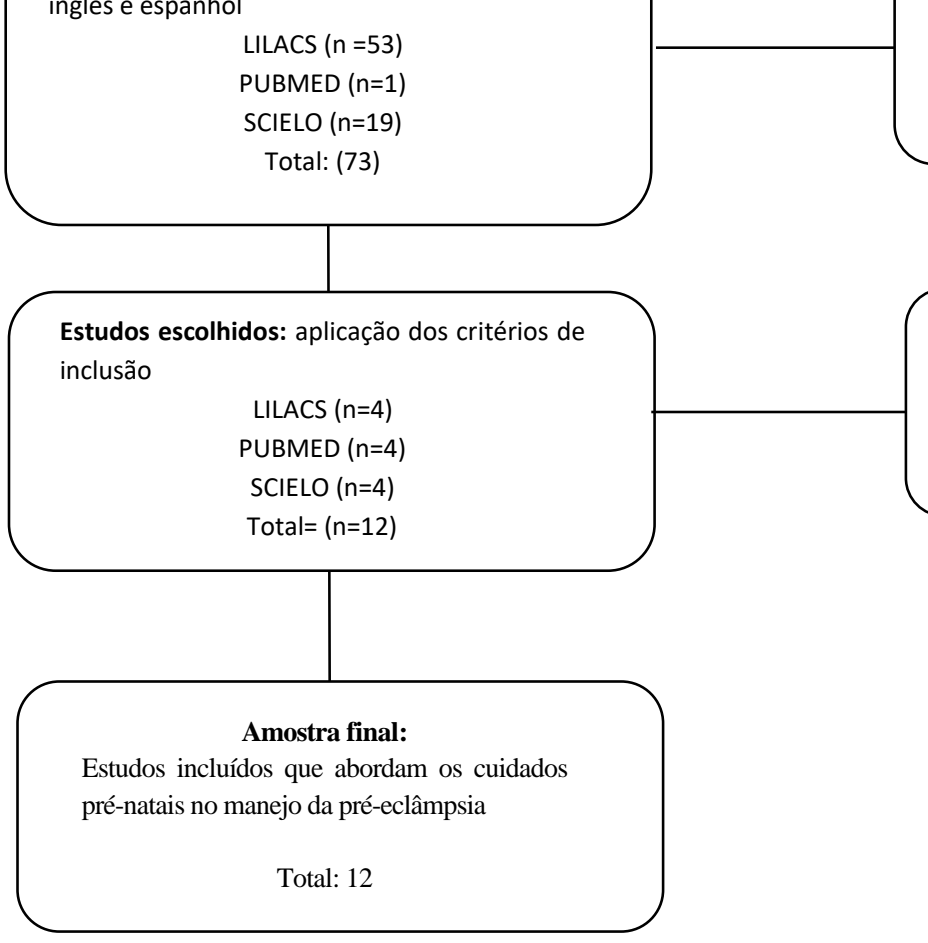

$\mathbf{1}^{\mathrm{a}}$ exclusão: Trabalhos que não possuem os filtros de ano de publicação e idioma

( $\mathrm{N}^{\mathrm{o}}$ de estudos excluídos:1.228) $2^{\text {a }}$ exclusão: Estudos excluídos a partir da leitura por não responder à pergunta norteadora do trabalho

( $\mathrm{N}^{\mathrm{o}}$ de estudos excluídos: 61)

Fonte: Autores.

\section{Resultados e Discussão}

A partir da análise dos estudos, baseado nos critérios de inclusão e que respondiam à pergunta norteadora "Quais os cuidados pré-natais no manejo da pré-eclâmpsia?", foram encontrados 12 artigos que estão evidenciados no Quadro 1, que estão dispostas as informações quando título, autores, países/ano, delineamento, objetivo e desfecho. Na disposição dos estudos foram encontrados 4 artigos no ano de 2017, 3 no ano de 2020, 2 de 2018 e 1 2021, em que 9 foram publicados em português, 2 em inglês 1 em espanhol, 9 eram pertencentes ao Brasil e 1 ao Reino Unido, França e Honduras.

No que diz respeito ao delineamento dos artigos, 4 eram uma revisão sistemática da literatura, 2 estudos descritivos, 2 caso-controle, 1 estudo transversal, 1 estudo ecológico, 1 estudo avaliativo e 1 estudo de meta-análise. 
Quadro 1 - Síntese dos artigos incluídos na revisão por título, ano de publicação, objetivo e delineamento.

\begin{tabular}{|c|c|c|c|c|c|}
\hline TÍTULO & AUTORES & $\begin{array}{l}\text { PAÍS / } \\
\text { ANO }\end{array}$ & DELINEAMENTO & OBJETIVO & DESFECHO \\
\hline $\begin{array}{l}\text { Pré-eclâmpsia pré-termo } \\
\text { e o melhor momento } \\
\text { para a resolução da } \\
\text { gestação: revisão } \\
\text { sistemática da literatura }\end{array}$ & $\begin{array}{lr}\text { GUIDA, J. P.S; } \\
\text { SURITA, F. G; } \\
\text { PARPINELLI, M. A; } \\
\text { COSTA, M. L. }\end{array}$ & $\begin{array}{c}\text { Brasil } \\
2017\end{array}$ & $\begin{array}{l}\text { Revisão Sistemática da } \\
\text { Literatura }\end{array}$ & $\begin{array}{lr}\text { Estabelecer } & \text { melhor } \\
\text { momento } & \text { de } \\
\text { resolução } & \text { da } \\
\text { gestação } & \text { em } \\
\text { mulheres com } & \text { pré- } \\
\text { eclâmpsia antes } & \text { das } \\
37 \text { semanas. } & \end{array}$ & $\begin{array}{l}\text { - Manejo expectante de } \\
\text { gestante cm PE, objetivando } \\
\text { a diminuição de } \\
\text { complicações perinatais; } \\
\text { - Análise da progressão da } \\
\text { pré-eclâmpsia. } \\
\text { - Destaca a indução do parto } \\
\text { prematuro, como alternativa } \\
\text { da cesariana evitando } \\
\text { desfechos obstétricos } \\
\text { negativos. }\end{array}$ \\
\hline $\begin{array}{l}\text { Cuidados pré-natais e } \\
\text { puerperais às gestantes } \\
\text { de um centro de saúde de } \\
\text { Minas Gerais quanto ao } \\
\text { risco de pré-eclâmpsia: } \\
\text { aspectos clínicos, } \\
\text { nutricionais } \\
\text { terapêuticos }\end{array}$ & 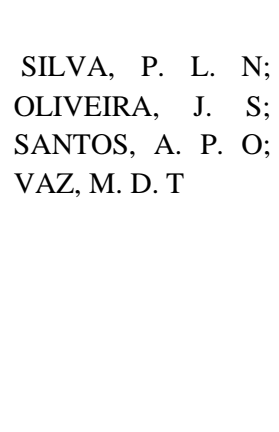 & $\begin{array}{c}\text { Brasil } \\
2017\end{array}$ & $\begin{array}{l}\text { Estudo descritivo, } \\
\text { exploratório, } \\
\text { documental, com } \\
\text { abordagem quantitativa }\end{array}$ & $\begin{array}{l}\text { Avaliar os aspectos } \\
\text { clínicos, nutricionais } \\
\mathrm{e} \text { terapêuticos } \\
\text { nos cuidados pré- } \\
\text { natais e puerperais } \\
\text { às gestantes de de } \\
\text { um centro quanto } \\
\text { saúde de Minas Gerai } \\
\mathrm{s} \text { a risco de pré- } \\
\text { ao râmpsia. }\end{array}$ & $\begin{array}{l}\text { - Análise do perfil } \\
\text { nutricional das gestantes } \\
\text { com PE refletindo seus } \\
\text { hábitos de vida; } \\
\text { - Observou-se o histórico } \\
\text { familiar e principais } \\
\text { características das gestantes } \\
\text { com PE. }\end{array}$ \\
\hline $\begin{array}{l}\text { Síndrome hipertensiva e } \\
\text { resultados perinatais em } \\
\text { gestação de alto risco }\end{array}$ & $\begin{array}{l}\text { ANTUNES, M.B; } \\
\text { DEMITTO, M. O; } \\
\text { GRAVENA, A. A. F; } \\
\text { PADOVANI, C; } \\
\text { PELLOSO, S. M. }\end{array}$ & $\begin{array}{c}\text { Brasil } \\
2017\end{array}$ & $\begin{array}{l}\text { Estudo do tipo caso- } \\
\text { controle, }\end{array}$ & $\begin{array}{l}\text { Analisar os } \\
\text { resultados perinatais } \\
\text { de gestantes de alto } \\
\text { risco com síndrome } \\
\text { hipertensiva. }\end{array}$ & $\begin{array}{l}\text {-Avaliação de prontuários } \\
\text { de gestante com PE de alto } \\
\text { risco; } \\
\text { - Verificou-se os principais } \\
\text { tipos de complicação } \\
\text { refente a oscilação da PA: } \\
\text { hipertensão crônica, pré- } \\
\text { eclâmpsia/eclâmpsia, } \\
\text { Síndrome de HELLP }\end{array}$ \\
\hline $\begin{array}{l}\text { Desfechos perinatais de } \\
\text { gestantes com pré- } \\
\text { eclampsia grave: Estudo } \\
\text { transversal }\end{array}$ & $\begin{array}{l}\text { CASSIANO, A. D. } \\
\text { N; VITORINO, A. } \\
\text { B. F; SILVA, M. D. } \\
\text { L. C. D. S; } \\
\text { NÓBREGA, C. S. M. } \\
\text { D. H; PINTO, E. S. } \\
\text { G. P; SOUZA, N. L. } \\
\text { D. S. }\end{array}$ & $\begin{array}{c}\text { Brasil } \\
2020\end{array}$ & $\begin{array}{l}\text { Estudo transversal e } \\
\text { descritivo }\end{array}$ & $\begin{array}{l}\text { Investigar } r \text { os } \\
\text { desfechos perinatais } \\
\text { de gestantes com } \\
\text { diagnóstico de pré- } \\
\text { eclâmpsia grave }\end{array}$ & $\begin{array}{l}\text {-Principais desfechos } \\
\text { perinatais em gestantes com } \\
\text { PEG; } \\
\text {-Características } \\
\text { socioeconômicas } \\
\text { obstétricas das gestantes } \\
\text { com diagnóstico de PEG. }\end{array}$ \\
\hline $\begin{array}{l}\text { Cuidados de enfermería } \\
\text { en pre-eclampsia leve en } \\
\text { el Hospital Mario } \\
\text { Catarino Rivas, I } \\
\text { semestre, } 2018\end{array}$ & $\begin{array}{ll}\text { MEJÍA, N. } & \text { L; } \\
\text { BAQUEDANO, } & \text { V. } \\
\text { M. M. } & \end{array}$ & $\begin{array}{c}\text { Hondura } \\
\text { s } \\
2018\end{array}$ & Estudo de caso & $\begin{array}{l}\text { Realizar um estudo } \\
\text { de caso de pré- } \\
\text { eclâmpsia leve. }\end{array}$ & $\begin{array}{l}\text {-Evidenciou-se déficit de } \\
\text { autocuidado, sentimento de } \\
\text { abandono, esperança } \\
\text { aumento da ansiedade }\end{array}$ \\
\hline $\begin{array}{l}\text { Aspectos relacionados às } \\
\text { internações por } \\
\text { intercorrências } \\
\text { gestacionais }\end{array}$ & $\begin{array}{l}\text { MEDEIROS, F. D. } \\
\text { D. A; SILVA, M. D. } \\
\text { G; SALES, J. C; } \\
\text { RIBEIRO, S. G; } \\
\text { JÚNIOR, F. J. G. D. } \\
\text { S; PARENTE, A. D. } \\
\text { C. M. }\end{array}$ & $\begin{array}{c}\text { Brasil } \\
2020\end{array}$ & $\begin{array}{l}\text { estudo de abordagem } \\
\text { quantitativa, descritivo } \\
\text { e retrospectivo. }\end{array}$ & $\begin{array}{lr}\text { Analisar aspectos } \\
\text { relacionados } & \text { às } \\
\text { internações } & \text { por } \\
\text { intercorrências } & \\
\text { gestacionais. } & \end{array}$ & $\begin{array}{l}\text {-Destaca as variáveis } \\
\text { sociodemográficas das mães } \\
\text { com PE. } \\
\text { - Fatores favoráveis para o } \\
\text { desenvolvimento da PE. }\end{array}$ \\
\hline
\end{tabular}




\begin{tabular}{|c|c|c|c|c|c|}
\hline Pre-eclampsia/Eclampsia & 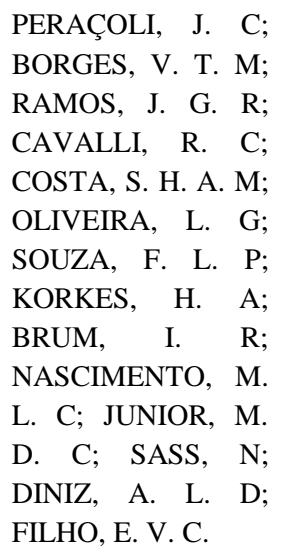 & $\begin{array}{l}\text { Brasil } \\
2021\end{array}$ & Revisão sistêmica & $\begin{array}{l}\text { Analisar os aspectos } \\
\text { de interesse prático } \\
\text { no atendimento } \\
\text { clínico e obstétrico } \\
\text { de mulheres com PE. }\end{array}$ & $\begin{array}{l}\text {-Explora etiologia, } \\
\text { fisiopatologia, diagnóstico, } \\
\text { resultados adversos } \\
\text { prevenção da PE }\end{array}$ \\
\hline $\begin{array}{l}\text { Avaliação do impacto de } \\
\text { programas de assistência } \\
\text { pré-natal, parto e ao } \\
\text { recém-nascido } \\
\text { mortes nas } \\
\text { evitáveis neonatais } \\
\text { Pernambuco, } \\
\text { estudo de adequação }\end{array}$ & $\begin{array}{lll}\text { LIMA, } & \text { S. } & \text { S; } \\
\text { BRAGA, } & \text { M. } & \text { C; } \\
\text { VANDERLEI, } & \text { L. } & \text { C. } \\
\text { M; LUNA, } & \text { C. } & \text { F; } \\
\text { FRIAS, P. G. } & \end{array}$ & $\begin{array}{l}\text { Brasil } \\
2020\end{array}$ & $\begin{array}{l}\text { Estudo ecológico, de } \\
\text { série temporal }\end{array}$ & $\begin{array}{l}\text { Avaliar o impacto de } \\
\text { programas voltados à } \\
\text { assistência pré-natal, } \\
\text { parto e ao recém- } \\
\text { nascido (Mãe Coruja } \\
\text { Pernambucana } \\
\text { Rede Cegonha) na } \\
\text { mortalidade neonatal } \\
\text { evitável no Estado de } \\
\text { Pernambuco, Brasil, } \\
\text { utilizando } \\
\text { abordagem } \\
\text { adequação }\end{array}$ & $\begin{array}{l}\text { - Importância da Rede } \\
\text { Cegonha; } \\
\text {-Importância de planos } \\
\text { assistenciais para a melhoria } \\
\text { da qualidade de assistência } \\
\text { à gestantes e crianças }\end{array}$ \\
\hline $\begin{array}{l}\text { Avaliação da Rede } \\
\text { Cegonha: devolutiva dos } \\
\text { resultados para as } \\
\text { maternidades no Brasil. }\end{array}$ & $\begin{array}{l}\text { SILVA, L. B. R. A. } \\
\text { A; TUESTA, A. A; } \\
\text { MASSARI, M. T. R; } \\
\text { AUSGUSTO, L. C. } \\
\text { R; GONÇALVES, L. } \\
\text { L. M; SILVA, C. K. } \\
\text { R. T; MINOIA, N. P }\end{array}$ & $\begin{array}{c}\text { Brasil } \\
2021\end{array}$ & Estudo avaliativo & $\begin{array}{l}\text { Descrever e avaliar o } \\
\text { processo de } \\
\text { devolutiva } \\
\text { resultados do } 2^{\circ} \text { ciclo } \\
\text { avaliativo de boas } \\
\text { práticas na atenção } \\
\text { ao parto } \\
\text { nascimento, em } \\
\text { maternidades } \\
\text { vinculadas à RC. }\end{array}$ & $\begin{array}{l}\text { - Importância e vantagens } \\
\text { da Rede Cegonha na } \\
\text { assistência a gestante }\end{array}$ \\
\hline $\begin{array}{l}\text { Pré-natal do parceiro: } \\
\text { desafios para o } \\
\text { enfermeiro }\end{array}$ & $\begin{array}{l}\text { SILVA, W. } \\
\text { WANDERLEY, } \\
\text { R; MARKUS, G. W. } \\
\text { S; PEREIRA, R. A; } \\
\text { COUTO, G. B. F; } \\
\text { DIAS, A. K }\end{array}$ & $\begin{array}{l}\text { Brasil } \\
2020\end{array}$ & $\begin{array}{l}\text { Revisão integrativa da } \\
\text { literatura com } \\
\text { abordagem qualitativa }\end{array}$ & $\begin{array}{l}\text { Identificar } \text { os } \\
\text { principais desafios } \\
\text { do enfermeiro no } \\
\text { pré-natal do parceiro }\end{array}$ & $\begin{array}{l}\text { - Dificuldades na adesão do } \\
\text { pré-natal } \\
\text { - Guia do Pré-natal do } \\
\text { parceiro }\end{array}$ \\
\hline $\begin{array}{l}\text { Aspirin for prevention of } \\
\text { preeclampsia }\end{array}$ & $\begin{array}{l}\text { ATALLAH, } \\
\text { LECARPENTIER, } \\
\text { E; GOFFINET, F; } \\
\text { DORET-DION, M; } \\
\text { GAUCHERAND, P; } \\
\text { TSATSARIS, V. }\end{array}$ & $\begin{array}{c}\text { França } \\
2017\end{array}$ & Estudo de meta-análise & $\begin{array}{lrr}\text { fornecer } & & \text { uma } \\
\text { atualização } & \text { sobre os } \\
\text { modos de } & \text { ação da } \\
\text { aspirina } & \text { e } & \text { suas } \\
\text { indicações } & & \text { na } \\
\text { prevenção } & \text { da } & \text { pré- } \\
\text { eclâmpsia } & & \end{array}$ & $\begin{array}{l}\text { - Diminuição da incidência } \\
\text { de PE e mortalidade } \\
\text { neonatal ao usar o AAS de } \\
\text { forma preventiva. }\end{array}$ \\
\hline $\begin{array}{l}\text { Calcium } \\
\text { supplementation during } \\
\text { pregnancy for preventing } \\
\text { hypertensive disorders } \\
\text { and related problems }\end{array}$ & 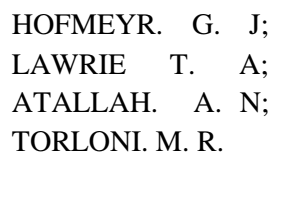 & $\begin{array}{l}\text { Reino } \\
\text { Unido } \\
2018\end{array}$ & Revisão de Literatura & $\begin{array}{lr}\text { Avaliar os efeitos da } \\
\text { suplementação de } \\
\text { cálcio durante } \\
\text { gravidez a } \\
\text { distúrbios } & \text { em } \\
\text { hipertensivos } & \end{array}$ & $\begin{array}{l}\text { - Redução da incidência de } \\
\text { síndromes hipertensivas ao } \\
\text { usar suplementação de } \\
\text { cálcio. }\end{array}$ \\
\hline
\end{tabular}

Fonte: Autores.

Vários autores relatam que a pré-eclâmpsia é a mais comum complicação e de maior mortalidade materna e fetal, afetando de 3 a $5 \%$ das mulheres gravida, seguida da síndrome de HELLP, eventos tromboembólicos, deslocamento de placenta e morte materna (Guida et al., 2017; Silva et al., 2017). Logo, a progressão da PE para forma grave ocorre em quase 
metade das mulheres (43\%), a PEG é diagnosticada mediante a pressão arterial de 160/110 mmHg e/ou eclampsia (Guida et al., 2017)

$\mathrm{Na}$ análise do estudo de Silva $e t$ al, verificou-se que a prevalência de gestantes com PE era de mulheres jovens com idade de 18 a 24 anos (52,7\%), primíparas (61,2\%), que não apresentavam histórico familiar de Síndromes Hipertensivas (SH) e 77,7\% das gestantes obtiveram oscilação na aferição de pressão arterial (PA). Ademais, 61,2\% dessas gestantes não faziam um acompanhamento pré-natal, fator esse que corrobora para um desfecho obstétrico negativo, uma vez que a oferta de uma assistência eficaz garante a identificação precoce da PE e outras SH, bem como seus agravos.

Com relação ao perfil socioeconômico das genitoras, a grande maioria possuía renda familiar por volta de 1 saláriomínimo e menos da metade possuía ensino médio completo (Cassiano et al., 2020; Medeiros et al., 2020). Esses dados mostram a importância da questão socioeconômica para a predisposição à fatores que estimulam a uma gravidez com maior risco de PE, visto que a renda e a escolaridade estão associadas ao maior abandono do pré-natal e a baixa informação.

No estudo, houve uma relação da PE com Óbitos Fetais Intrauterinos (OFIU) e Óbitos Neonatais Precoce (ONP), uma vez que esse índice de mortalidade era de 7,6\% para OFIU e 1,3\% de ONP (Cassiano et al., 2020). Dessa forma, é possível salientar que a PE é um fato de risco para o aumento da mortalidade, principalmente intrauterino. Essas complicações após o nascimento são recorrentes devido a alterações no desenvolvimento placentário, pois a vasoconstricção materna restringe o envio de nutrientes e de oxigênio, o que prejudica o desenvolvimento fetal.

Nesse sentido, o Ministério da Saúde (MS) instituiu a Rede Cegonha (RC), mediante a Portaria GM/MS 1.459 de 2011, objetivando a melhoria da qualidade da assistência integral à saúde da mulher e da criança, a queda dos índices de morbimortalidade materna e neonatal e a melhoria no acesso aos cuidados pré-natais, assistência ao parto e puerpério e ao cuidado com à criança até 24 meses de vida. (Lima et al., 2020; Silva et al., 2021). À vista disso, a RC configura-se como uma das principais e mais importante estratégia da rede de assistência que vem assegurar o acesso e acolhimento da gestante e da criança, além de uma assistência mais humanizada.

Mediante a análise dos estudos, evidencia-se que a taxa de mortalidade materno-infantil no Brasil atinge cerca de 140 óbitos por 100.000 nascidos vivos. Sendo assim, essa estatística reflete as principais causas de óbitos maternos que dentre elas são infecções, pré-eclâmpsia, intervenções obstétricas desnecessárias, hemorragias, descolamento de placenta (Marques et al., 2020). Dessa forma, o pré-natal adequado garante ações de prevenção, promoção e tratamento que visam a redução de desfechos obstétricos desfavoráveis e promovem condutas efetivas para a redução da mortalidade materna e infantil.

Outrossim, de acordo com dados de Cassiano et al, os nascidos vivos, por causa também da vasoconstrição materna, possuíam maior grau de complicações nos primeiros minutos de vida, uma vez que 48,4\% nasceram com idade pré-termo, e $56,7 \%$ nasceram com baixo peso (menos de 2.500g), já o índice APGAR acusou anóxia moderada em 21\% dos recém-nascidos no primeiro minuto e $11,5 \%$ no quinto minuto de vida. Nesse modo, a boa adesão ao pré-natal é primordial para contrapor a essa estatística por conta do monitoramento fetal constante, o que permite avaliar a pressão arterial, peso e altura do feto de acordo com a semana gestacional. Nesse cenário evidencia-se a importância da atuação precoce dos profissionais da saúde para a reversão de quadros indesejáveis.

Em consideração a isso, dados dos estudos observados enfatizam a importância do uso precoce de baixas doses de ácido acetilsalicílico (AAS) aliada à suplementação de cálcio, pois reduz significativamente o risco de desenvolvimento da PE em gravidez de médio ou alto risco de desenvolvimento de síndromes hipertensivas de 8 a 10\% (Atallah et al., 2017; Hofmyer et al., 2018). À vista disso, é importante enfatizar a importância de iniciar o pré-natal excessivamente nos primeiros meses de gestação, pois o diagnóstico precoce dessa síndrome hipertensiva pode ser evitado mais facilmente ao ponto de diminuir as taxas de mortalidade perinatal do que diagnosticada tardiamente. (Guida et al., 2017). 
Recorrentemente, o estresse é um fator muito associado ao desenvolvimento da PE, dado que a exposição a esses fatores pode estimular ao aumento sistêmico da pressão sanguínea. Diante disso, mesmo com a maioria das gestantes dos casos estudados possuírem um conjugue, o sentimento de abandono, ansiedade e perda de esperança são recorrentes em virtude da ausência da figura paterna no decorrer da gestação, o que é importante para a diminuição da evasão materna no pré-natal (Medeiros et al., 2020; Mejia \& Baquedano, 2018;). Sendo assim, é fundamental no momento da assistência, analisar a questão psicológica da gestante, observando a expressão facial, estado de ânimo e como está se sentindo naquele momento, além de estimular o acompanhamento paterno no pré-natal. (Mejia \& Baquedano, 2018; Peraçolli et al., 2019).

Mediante isso, é de suma importância a inclusão e participação ativa do parceiro durante todo o planejamento reprodutivo, como forma de garantir a criação e fortalecimento de vínculos não apenas com a sua parceira mais com seu filho, criando uma atmosfera familiar saudável. No entanto, muitos obstáculos, resistências e desafios são enfrentados pela equipe de saúde no processo de inclusão do parceiro no pré-natal.

Dessa forma, o MS criou em 2016 o Guia do Pré-natal do Parceiro que inclui ações às quais os profissionais de saúde devem adotar para envolver o homem de forma integral no processo gestacional e puerperal. Ademais, os estudos evidenciam resultados positivos da presença ativa do homem em todo processo gestacional, desde o pré-natal auxiliando na adesão da mulher ao acompanhamento até o momento do parto onde a sua participação promove alívio de dor, pode diminuir o tempo de trabalho de parto e por fim auxiliando na diminuição da probabilidade da mulher desenvolver depressão pós-parto (Silva et al., 2020).

Nesse sentido, a anamnese e a triagem realizada na primeira consulta pré-natal são essenciais para traçar ações de cuidado, priorizando as reais necessidades da gestante com PE, visando as possíveis reduções de danos. Portanto, é necessário que o profissional responsável por esse momento busque informações acerca do histórico de PE em mulheres multíparas, conheça os padrões de PA da gestante, a presença de patologias pré-existentes ou fatores de riscos, como cor, idade, obesidade, diabetes mellitus, estresse e entre outros (Peraçolli et al., 2021)

Estudos relatam, que o risco de PE na primeira gestação é de 4,1\% e 1,7\% nas gestações seguintes (Antunes et al., 2017). Diante desses achados, evidencia-se a importância do conhecimento acerca do histórico obstétrico, para que possa incluir para a gestante uma assistência Pré-natal de Alto Risco (PNAR) que irá fornecer um acompanhamento especializado durante todo o período gestacional.

No que diz respeito ao conhecimento das gestantes sobre a PE, os estudos relatam que o conhecimento é limitado, o que reflete negativamente na qualidade da assistência fornecida (Silva et al., 2017). Contudo, é imprescindível que durante todo o processo gestacional a mulher tenha acesso as informações sobre a patologia, para que juntamente com a equipe multiprofissional venha desenvolver o planejamento reprodutivo com menor risco de desfechos desfavoráveis.

Dessa maneira, cabe aos profissionais da saúde o papel de sensibilizar e informar as gestantes sobre os riscos, cuidados, uso de medicação, alimentação, orientações sobre as consultas, além de manter uma comunicação direta e ativa durante toda a assistência. Outra linha de análise, foi o perfil nutricional das gestantes em que $8,5 \%$ apresentavam uma alimentação com restrição de sal e 16,7\% faziam quatros refeições durante o dia (Silva et al., 2017).

Em vista disso, durante o PNAR a presença da equipe multiprofissional - nutricionista, enfermeiro, médico, educador físico - vai garantir um cuidado completo, de forma que a gestante seja assistida por diferentes profissionais da área que irá contribuir para a melhoria dos hábitos e da qualidade gestacional (Antunes et al., 2017). Além disso, fornecer à gestante o acesso a todos os níveis de assistência durantes os cuidados pré-natais. 


\section{Considerações Finais}

Com o presente trabalho observa-se a importância de uma boa assistência pré-natal mediante os riscos associados à PE. Uma vez que há a associação da PA materna e intrauterina elevadas com o aumento da mortalidade materna, do OFIU e do ONP e do comprometimento do crescimento do recém-nascidos de mães com PE.

Por outro lado, é importante o fornecimento do apoio psicológico nesse período de desenvolvendo fetal, além do aconselhamento da figura paterna no que tange o acompanhamento da gestação, visto que houve uma relação desse fator com o aumento da incidência de PE e da dificuldade de adesão ao pré-natal.

Paralelamente, é possível destacar o papel do governo no que diz respeito ao fortalecimento de assistências sociais à renda e ao incentivo ao desenvolvimento escolar, posto que foi encontrado similaridades do perfil socioeconômico das genitoras com o abandono do pré-natal e aumento da incidência da PE, sendo que mulheres com baixa renda familiar e baixa escolaridade possuem maior tendência a evasão do programa.

Não obstante, faz-se necessário que a gestante tenha um acompanhamento multiprofissional durante a sua assistência pré-natal, visando um atendimento holístico que supra as suas necessidades nutricionais, físicas e clínicas durante todo o período gestacional. Cabe ainda a esses profissionais, o repasse de informações acerca da PE de maneira a manter a gestante informada quando a prevenção, tratamento e cuidados necessário durante esse período. Ademais, é necessário proporcionar à mulher uma participação ativa no seu pré-natal de maneira a traçar medidas factíveis a sua realidade e que englobem todas as suas reais necessidades.

Evidencia-se ainda, a necessidade de literaturas que abordem o manejo das gestantes com PE no pré-natal, uma vez que, não foram encontradas muitas produções que abordassem a temática. Diante disso, é importante discutir os cuidados prénatais no manejo da PE, visto que é uma patologia muito recorrente nas gestantes, a fim de criar um conhecimento e cuidado humanizado.

\section{Referências}

Antunes, M. B., Demitto, M. O., Gravena, A. A. F., Padovani, C., \& Pelloso, S. M. (2017). Síndrome hipertensiva e resultados perinatais em gestação de alto risco. Revista Mineira de Enfermagem, v. 21, p. 1-6.

Atallah, A., Lecarpentier, E., Goffinet, F., Doret-dion, M, Gaucherand, P., \& Tsatsaris, V. (2017) Aspirin for Prevention of Preeclampsia. Drugs. Nov;77(17):1819-1831. 10.1007/s40265-017-0823-0. PMID: 29039130; PMCID: PMC5681618.

Cassiano, A. D. N., Vitorino, A. B. F., Silva, M. D. L. C. D. S., Nóbrega, C. S. M. D. H., Pinto, E. S. G. P., \& Souza, N. L. D. S. (2019). Desfechos perinatais de gestantes com pré-eclampsia grave: estudo transversal. Online braz. j. nurs.(Online).

SUS - DATASUS. (2021) Informações de Saúde, Estatísticas Vitais: banco de dados. http://tabnet.datasus.gov.br/cgi/tabcgi.exe?sim/cnv/mat10uf.def>.

Fassarella, B. P. A., Almeida, G., Teles, D. A., Ortiz, L. S., Silva, I. S., Nezes, K. C., Costa, P. A. F. S., \& Evangelista, D. S. (2020) Cuidados de enfermagem direcionados à gestante portadora de doença hipertensiva específica da gravidez. Research, Society and Development, 9(9), e343996768-e343996768.

Federação Brasileira das Associações de Ginecologia e Obstetrícia (FEBRASGO). (2019). Pré-eclâmpsia. Femina. 7(5).

Fundação Oswaldo Cruz. (2018). Profilaxia da pré-eclâmpsia no pré-natal. Portal de Boas Práticas em Saúde da Mulher, da Criança e do Adolescente. https://portaldeboaspraticas.iff.fiocruz.br/atencao-mulher/profilaxia-da-pre-eclampsia-no-pre-natal/>.

Guida, J. P. S., Surita, F. G., Parpinelli, M. A., \& Costa, M. L. (2017). Pré-eclâmpsia pré-termo e o melhor momento para a resolução da gestação: revisão sistemática da literatura. Revista Brasileira de Ginecologia e Obstetrícia, 39(11), 622-631.

Guida, J. P. S. (2017). Pré-Eclâmpsia: Entender Para Aprimorar O Cuidado. Tese (Mestrado em Ciências da Saúde) - Faculdade de Ciências Médicas, Universidade Estadual de Campinas; Campinas; p. 153.

Gonçalves, J. R. (2019) Como escrever um Artigo de Revisão de Literatura. Revista JRG de Estudos Acadêmicos, 2(5), $29-55$.

Governo de Santa Catarina. (2019). A pré-eclâmpsia afeta té 7\% das brasileiras. Secretaria de Saúde. https://www.saude.sc.gov.br/index.php/noticiasgeral/todas-as-noticias/1641-noticias-2019/10661-a-pre-eclampsia-afeta-ate-7-das-brasileiras〉.

Hofmeyr. G. J., Lawriet T. A., Atallah. A. N., \& Torloni. M. R. (2018). Calcium supplementation during pregnancy for preventing hypertensive disorders and related problems. Cochrane Database Syst Rev. Oct 1;10(10):CD001059. 10.1002/14651858.CD001059.pub5. PMID: 30277579; PMCID: PMC6517256. 
Kahhale, S., Francisco, R. P. V., \& Zuhaib, M. (2018). Pré-eclâmpsia. Revista de Medicina, 97(2), $226-234$.

Lima, S. S., Braga, M. C., Vanderlei, L. C. M., Luna, C. F., \& Frias, P. G. (2020). Avaliação do impacto de programas de assistência pré-natal, parto e ao recém-nascido nas mortes neonatais evitáveis em Pernambuco, Brasil: estudo de adequação. Cadernos de Saúde Pública, v. 36.

Marques, B. L., Tomasi, Y. T., Saraiva, S. S., Boing, A. F., \& Geremia, D. S. (2020). Orientações às gestantes no pré-natal: a importância do cuidado compartilhado na atenção primária em saúdea. Escola Anna Nery, v. 25.

Marques, R. A., Marder, J., Bertão, S. V. R., Silva, V. C., Souza, G. L., \& Konnopka, C. K. (2020). Doença Hipertensiva Gestacional: A Importância Do PréNatal Para Prevenir Desfechos Desfavoráveis. Anais do Salão Internacional de Ensino, Pesquisa e Extensão, 12(2).

Medeiros, F. D. D. A., Silva, M. D. G., Sales, J. C., Ribeiro, S. G., Júnior, F. J. G. D. S., \& Parente, A. D. C. M. (2020). Aspectos relacionados às internações por intercorrências gestacionais. Enferm. foco (Brasília), p. 41-48.

Mejia, N. L., \& Baquedano, V. M. M. (2018). Cuidados de enfermería en pre-eclampsia leve en el Hospital Mario Catarino Rivas, I semestre, 2018. Revista Científica de la Escuela Universitaria de las Ciencias de la Salud, 5(2), 32-41.

Mendes. R. B., Santos, J. M. J., Prado, D. S., Gurgel, R. Q., Bezerra, F. D., \& Gurgel, R. Q. (2020). Avaliação da qualidade do pré-natal a partir das recomendações do Programa de Humanização no Pré-natal e Nascimento. Ciência \& Saúde Coletiva, 25, $793-804$.

Nascimento, D. S., Nascimento, D. S., Silva, V. F. A., Belarmino, C. M. V., \& Lago, V. C. A. L. P. (2021). Assistência de enfermagem ao pré-natal na atenção básica: uma revisão integrativa. Revista Artigos. Com, v. 27, p. e7219-e7219.

Oldra, L. C., Oliveira, D. L., Campos, G. S., Firmino, I. R., Garcia, L. A., \& Peres, P. M. (2021). A saúde da mulher após um quadro de pré-eclâmpsia: uma revisão de literatura. Brazilian Journal of Development, 7(2), 17281-17284.

Oliveira, R. L., Bernadelli, M., Santos, C. P., Almeira, S. K. R., Souza, L. A. C. F., Almeida, B. S., Reis, L. D., Barros, R. R., Cardoso, J. S., \& Libe, F. M. P. Atenção ao Pré-natal de alto risco e o manejo por profissionais da Estratégia de Saúde da Família: um relato de experiência profissional. Brazilian Journal of Health Review, 4(1), 2021.

Organização Mundial da Saúde (OMS). Evidências cientificas dos dez passos para o sucesso do aleitamento materno. [Tradução de Maria Cristina Gomes do Monte]. Brasília (DF): OPAS; 2001.

Peraçoli, J. C., Borgues, V. T. M., Ramos, J. G. R., Cavalli, R. C., Costa, S. H. A. M., Oliveira, L. G., Souza, F. L. P., Korkes, H. A., Brum, I. R., Nascimento, M. L. C., Junior, M. D. C., Sass, N., Diniz, A. L. D., \& Filho, E. V. C. (2019). Pre-eclampsia/Eclampsia. Revista Brasileira de Ginecologia e Obstetrícia.

Santos, C. R., \& Batista, F. M. A. (2018). Assistência De Enfermagem À Gestante Com Pré-Eclâmpsia Durante O Pré-Natal. Trabalho de Conclusão de Curso de Enfermagem - Universidade Federal do Piauí.

Silva, D. A. (2020). Cuidado ao pré-natal segundo indicadores do programa de humanização do pré-natal e nascimento. Rev. enferm. atenção saúde, p. 111123.

Silva, L. B. R. A. A., Tuesta, A. A., Massari, M. T. R., Ausgusto, L. C. R., Gonçalvez, L. L. M., Silva, C. K. R. T., \& Minoia, N. P. (2021). Avaliação da Rede Cegonha: devolutiva dos resultados para as maternidades no Brasil. Ciência \& Saúde Coletiva, v. 26, p. 931-940.

Silva, N. M. C., Landini, C. L. S., Lisboa, S. C. B., \& Maganha, C. A. (2020). O Uso Do Ácido Acetil Salicílico Como Destaque Entre As Principais Terapêuticas Na Prevenção De Pré-Eclâmpsia. Equipe Editorial. 2(2), 126-131.

Silva, P. L. N., Oliveira, J. S., Santos, A. P. O., \& Vaz, M. D. T. (2017). Cuidados pré-natais e puerperais às gestantes de um centro de saúde de Minas Gerais quanto ao risco de pré-eclâmpsia: aspectos clínicos, nutricionais e terapêuticos. Journal of Health \& Biological Sciences, 5(4), 346-351.

Silva, W. C., Wanderley, R. R., Markus, G. W. S., Pereira, R. A., Couto, G. B. F., \& Dias, A. K. (2020). Pré-Natal Do Parceiro: Desafios Para O Enfermeiro. Revista Extensão, 4(2), 127-137.

Sociedade Brasileira de Cardiologia; Sociedade Brasileira de Hipertensão; Sociedade Brasileira de Nefrologia. (2021) VII Diretrizes Brasileiras de Hipertensão. Arq Bras Cardiol. 